\title{
New extended interpolating operators for hadron correlation functions
}

\section{Francesco Scardino*}

Dipartimento di Fisica, "Sapienza" Università di Roma, and INFN, Sezione di Roma, Piazzale Aldo Moro 2, I-00185 Roma, ITALY.

E-mail: francesco.scardino@romal.infn.it

\section{Mauro Papinutto}

Dipartimento di Fisica, "Sapienza" Università di Roma, and INFN, Sezione di Roma, Piazzale Aldo Moro 2, I-00185 Roma, ITALY.

E-mail: mauro.papinutto@roma1.infn.it

\section{Stefan Schaefer}

Neumann Institute for Computing, DESY, Platanenallee 6, 15738 Zeuthen, Germany

E-mail: stefan.schaeferdesy.de

\begin{abstract}
New extended interpolating operators made of quenched three dimensional fermions are introduced in the context of lattice QCD. The mass of the 3D fermions can be tuned in a controlled way to find a better overlap of the extended operators with the states of interest. The extended operators have good renormalisation properties and are easy to control when taking the continuum limit. Moreover the short distance behaviour of the two point functions built from these operators is greatly improved. The operators have been numerically implemented and a comparison to point sources and Jacobi smeared sources has been performed on the new CLS configurations.
\end{abstract}

34th annual International Symposium on Lattice Field Theory

24-30 July 2016

University of Southampton, UK

${ }^{*}$ Speaker. 


\section{Introduction}

A serious problem which limits the attainable precision in lattice QCD computations of hadronic observables is the exponential suppression of the signal-to-noise ratio in Euclidean time. It is therefore important to reduce the coefficient of this deterioration as much as possible.

One first step is to find improved interpolating operators, which allow to decrease statistical and systematic errors in the extraction of the hadron spectrum, form factors and matrix elements. For this purpose, we introduce extended interpolating operators built from 3D fermions. These are well behaved under renormalisation and improve the short distance behaviour of two point functions. The octet and decuplet baryonic interpolating operators have been classified according to the irreducible representations of the cubic group [1] [2]. This classification has the advantage of being exhaustive by construction.

In this proceedings contribution we first review the construction of the basis of baryon operators, before introducing the new source operators. They are then compared to the traditional Jacobi smearing.

\section{Classification of baryonic operators}

Baryons are bound states of three valence quarks. The interpolating operators $\mathscr{B}$ will need to have a well defined set of quantum numbers such that the corresponding Hilbert space operator $\hat{\mathscr{B}}$ projects onto the state we are interested in. On the lattice, rotational symmetry breaks down and is replaced by the cubic group $S O(3, \mathbb{Z})$ whose elements are the matrices of $S O(3)$ with integer entries. In order to correctly classify the operators, we will use the spin covering group $\operatorname{Spin}(3, \mathbb{Z})$ of $S O(3, \mathbb{Z})$. In fact the $\operatorname{Spin}(3)$ group is isomorphic to $S U(2)$ and allows us to work with the continuum Weyl spinor notation on the lattice.

A generic gauge invariant three-quark operator will have the form

$$
\mathscr{B}(x)=u_{\alpha}^{a}(x) d_{\beta}^{b}(x) s_{\gamma}^{c}(x) t^{\alpha \beta \gamma} \varepsilon_{a b c},
$$

where $u, d, s$ are the quark fields, which are in the fundamental representation of $S U(3)_{c}$. Furthermore, despite the notation we use, the fields have a definite but not necessarily different flavour. The tensor $t^{\alpha \beta \gamma}$ depends on the $S O(3, \mathbb{Z})$ representation the baryon falls in, $\varepsilon_{a b c}$ is the only allowed invariant colour tensor. Greek indices $\alpha, \beta, \ldots$ are Dirac indices while the Latin ones $(a, b, \ldots)$ represent colour.

In order to classify the baryonic operators according to the irreducible representations of flavour and the rotation group on the lattice, we need to classify the tensors $t^{\alpha \beta \gamma}$ introduced above according to the irreducible representations of the spin covering $\operatorname{Spin}(3, \mathbb{Z})$ of the cubic group $S O(3, \mathbb{Z})$. For spin $\frac{1}{2}$ and spin $\frac{3}{2}$ baryons, it can be proven [3] that there is a one to one correspondence between the corresponding $\operatorname{Spin}(3, \mathbb{Z})$ representations on the lattice and the continuum ones. We thus use the continuum notation of dotted and undotted Weyl spinors in the following.

We now discuss the operators that have been actually used in the simulations. Consider the case in which two flavours are equal, namely for spin $s=\frac{1}{2}$ the case of the nucleon. It is possible to show 
that there are two proton operators $\hat{p}$ and $\tilde{p}$ in the $\left(\frac{1}{2}, 0\right)$ representation

$$
\begin{array}{ll}
\hat{p}_{\frac{1}{2}, \frac{1}{2}}^{\left(\frac{1}{2}, 0\right)}=\frac{1}{\sqrt{2}}\left(u_{1} d_{2}-u_{2} d_{1}\right) u_{1} & \hat{p}_{\frac{1}{2},-\frac{1}{2}}^{\left(\frac{1}{2}, 0\right)}=\frac{1}{\sqrt{2}}\left(u_{1} d_{2}-u_{2} d_{1}\right) u_{2} \\
\tilde{p}_{\frac{1}{2}, \frac{1}{2}}^{\left(\frac{1}{2}, 0\right)}=\frac{1}{\sqrt{2}}\left(u_{\mathrm{i}} d_{\dot{2}}-u_{\dot{2}} d_{\mathrm{i}}\right) u_{1} & \tilde{p}_{\frac{1}{2},-\frac{1}{2}}^{\left(\frac{1}{2}, 0\right)}=\frac{1}{\sqrt{2}}\left(u_{\mathrm{i}} d_{2}-u_{\dot{2}} d_{\mathrm{i}}\right) u_{2}
\end{array}
$$

and one in the $\left(\frac{1}{2}, 1\right)$ representation

$$
\begin{aligned}
& p_{\frac{1}{2}, \frac{1}{2}}^{\left(\frac{1}{2}, 1\right)}=\frac{1}{\sqrt{14}}\left\{\left(u_{\mathrm{i}} d_{\dot{2}}+u_{\dot{2}} d_{\mathrm{i}}\right) u_{1}-2 u_{\mathrm{i}} d_{\mathrm{i}} u_{2}+2\left(u_{\mathrm{i}} d_{2}-u_{\mathrm{i}} d_{1}\right) u_{\mathrm{i}}\right\} \\
& p_{\frac{1}{2},-\frac{1}{2}}^{\left(\frac{1}{2}, 1\right)}=-\frac{1}{\sqrt{14}}\left\{\left(u_{\mathrm{i}} d_{\dot{2}}+u_{\mathrm{i}} d_{\mathrm{i}}\right) u_{2}-2 u_{\dot{2}} d_{\dot{2}} u_{1}+2\left(u_{\dot{2}} d_{1}-u_{\mathrm{i}} d_{2}\right) u_{\dot{2}}\right\} .
\end{aligned}
$$

In the case of three equal flavours, namely the case of the $\Omega$ baryon, there are two $s=\frac{3}{2}$ decouplet operators

$$
\begin{array}{ll}
\Omega_{\frac{3}{2}, \frac{3}{2}}^{\left(\frac{1}{2}, 1\right)}=s_{\mathrm{i}} s_{1} s_{1} & \Omega_{\frac{3}{2}, \frac{1}{2}}^{\left(\frac{1}{2}, 1\right)}=\frac{1}{\sqrt{3}}\left\{s_{1} s_{1} s_{2}+s_{1} s_{2} s_{1}+s_{2} s_{1} s_{1}\right\} \\
\Omega_{\frac{3}{2}, \frac{3}{2}}^{\left(\frac{3}{2}, 0\right)}=s_{1} s_{1} s_{1} & \Omega_{\frac{3}{2}, \frac{1}{2}}^{\left(\frac{3}{2}, 0\right)}=\frac{1}{\sqrt{3}}\left\{s_{1} s_{1} s_{2}+s_{1} s_{2} s_{1}+s_{2} s_{1} s_{1}\right\},
\end{array}
$$

where we have reported only the spin up components. In order to have operators with definite transformation properties under the action of parity $\hat{\mathscr{P}}$, we need to consider

$$
\mathscr{B}_{(a, b) \oplus(b, a)}^{ \pm}=\mathscr{B}_{(a, b)} \mp \mathscr{B}_{(b, a)},
$$

where \pm indicates the parity eigenvalue and $(a, b)$ the irreducible representation.

\section{Extended operators}

The 3D extended operators are built from quenched three dimensional fermions fields coupled via pseudoscalar bilinears with ordinary four dimensional fermions in the bulk. These 3D fields live on a time-slice and their propagator can be derived from the action

$$
S^{3 D}=a^{3} \sum_{\mathbf{x}} \bar{\varphi}(\mathbf{x}) \mathscr{D} \varphi(\mathbf{x}), \quad \mathscr{D}=\frac{1}{2} \sum_{i=1}^{3}\left\{\gamma_{i}\left(\nabla_{i}^{*}+\nabla_{i}\right)-a \nabla_{i}^{*} \nabla_{i}\right\}+m_{3 D}
$$

where $\mathscr{D}$ is the three dimensional Wilson-Dirac operator with a mass term $m_{3 D}$. The $3 \mathrm{D}$ fermionic fields $\varphi, \bar{\varphi}$ are spin $1 / 2$ spinors with canonical dimension 1 and represent a flavour $S U(3)$ triplet $\bar{\varphi}=(\overline{\mathbf{u}}, \overline{\mathbf{d}}, \overline{\mathbf{s}})$ which corresponds to the four dimensional triplet $\bar{\psi}=(\bar{u}, \bar{d}, \bar{s})$.

These fields are used to build an interpolating operator $\mathbf{B}$ with the same spin and flavour structure of the corresponding 4D local operators $\mathscr{B}(x)$ which we have defined above. This new operator is coupled with pseudoscalar bilinears of the form $\bar{\varphi} \gamma_{5} \psi$. Computations using baryonic two point functions have in fact shown that the signal obtained with the pseudoscalar bilinears has much lower noise contributions than the one given by the scalar bilinears. The coupling of $\mathbf{B}$ with bilinears allows the quenched three dimensional fermions to propagate in time via the four dimensional ones. 

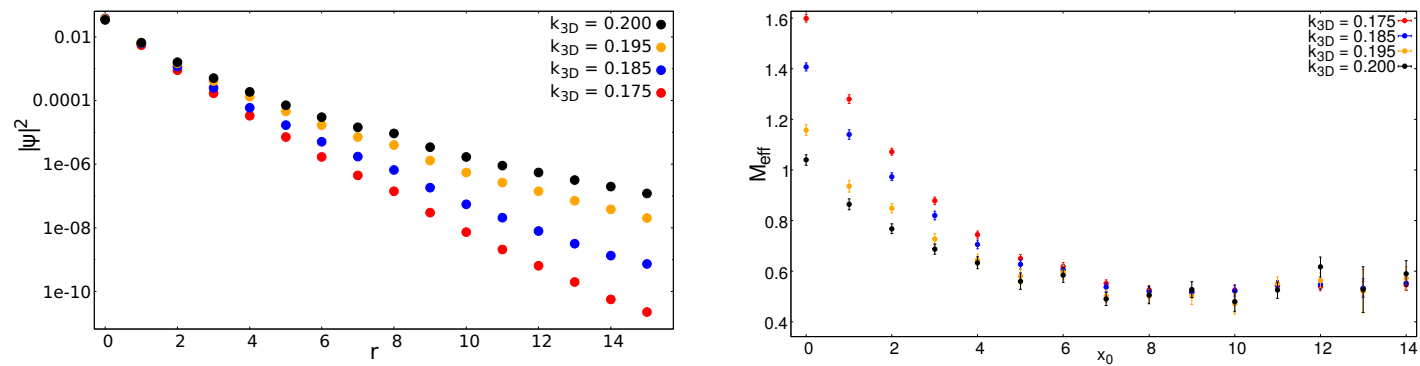

Figure 1: Left: Norm square of the 3D source $|\Psi|^{2}$ along one axis as a function of distance for the extended operators. For $\kappa_{3 D} \rightarrow \kappa_{3 D}^{c r i t}$ the $3 \mathrm{D}$ fields are much more spread out. Right: Effective mass of the nucleon for various values of $\kappa_{3 D}$. The larger $\kappa_{3 D}$, the stronger the excited states are suppressed.

It is now possible to join together the pieces and define the 3D extended operators

$$
O(t, \mathbf{w})=a^{9} \sum_{\mathbf{x}, \mathbf{y}, \mathbf{z}} \mathbf{B}(\mathbf{w}) \bar{\varphi} \gamma_{5} \psi(t, \mathbf{x}) \bar{\varphi} \gamma_{5} \psi(t, \mathbf{y}) \bar{\varphi} \gamma_{5} \psi(t, \mathbf{z}) .
$$

There are as many bilinear operators as there are quarks in the interpolating operator $\mathbf{B}$, for instance if we were to study mesons there would have been only two bilinears instead of three. Due to the fact that the 3D fermion action is symmetric under $C, P$ and $\Gamma$ symmetry ${ }^{1}$ it can be shown that the 3D extended operators are well behaved under renormalisation.

From the operator of Eq. (3.2), the smoothed baryonic two point functions are constructed in the usual way from products of quark propagators. For the 3D extended operators, this amounts to replacing each $4 \mathrm{D}$ propagator in the formula for the point sources with the product

$$
S\left(t, \mathbf{w}, t^{\prime}, \mathbf{w}^{\prime}\right)=a^{6} \sum_{\mathbf{x}, \mathbf{x}^{\prime}} S_{3 D}\left(\mathbf{w}, \mathbf{x}^{\prime}\right) \gamma_{5} S_{4 D}\left(t, \mathbf{x}^{\prime} ; t^{\prime}, \mathbf{x}\right) \gamma_{5} S_{3 D}\left(\mathbf{x} ; \mathbf{w}^{\prime}\right) .
$$

It can be shown from dimensional considerations, that the 3D extended operator's two point function is much more regular at short distances than the one where local operators are used. Indeed the degree of divergence in time will be at most logarithmic, while for the local operators it is a polynomial of degree six.

\section{Numerical tests}

The new operators have been numerically implemented and compared to point sources and Jacobi smearing. The lattice simulations were performed on the CLS $N_{f}=2+1$ gauge configurations [4] that adopt the open boundary conditions in time [5]. In the present exploratory study we have a flavour $S U(3)$ symmetric $96 \times 32^{3}$ ensemble with $M_{\pi}=M_{K}=420 \mathrm{MeV}$ and a lattice spacing $a=0.086 \mathrm{fm}[6]$.

\subsection{Shape of the source}

For this first test, we use the same 3D mass for all fermion fields, defining analogously to the $4 \mathrm{D}$ case $m_{3 D}=\frac{1}{2}\left(\frac{1}{\kappa_{3 D}}-6\right)$. The norm of the wave function as a function of the separation from the

\footnotetext{
${ }^{1} \varphi \rightarrow e^{i \alpha \Gamma} \varphi$ and $U_{\mu}(x) \rightarrow U_{\mu}(x)$ where $\Gamma=i \gamma_{0} \gamma_{5}$
} 




Figure 2: The norm of the 3D source (red) and the Jacobi smeared source (blue) as a function of distance $r$ for the extended operators. The parameters of the Jacobi smearing were tuned to match the average radii $\left\langle r^{2}\right\rangle$.

source is shown for select values of $\kappa_{3 D}$ in Fig. 1. As expected, a smaller 3D mass corresponds to a source which is more spread out, with the square of the effective mass being proportional to the 3D mass in a wide range.

For our value of the physical parameters, a critical value of $\kappa_{3 D}$ can be found, at which the effective mass of the three-dimensional analogue to the pion goes to zero. A linear fit to this behavior leads to an estimate of $\kappa_{3 D}^{\text {crit }}=0.208 \pm 0.004$ for this ensemble. For the rest of the study, we employed $\kappa_{3 D}=0.185$, which we have chosen to match standard parameters of Jacobi sources as explained below.

In the right hand plot of Fig. 1, the effect of the extension of the source on the nucleon two-point function is shown. The smaller $m_{3 D}$, i.e. the wider the source, the stronger is the suppression of the excited state contribution at small distances. Nevertheless, the plateau region from which the ground state mass is extracted, starts roughly at the same time separation.

\subsection{Comparison to Jacobi smearing}

To test the effectiveness of the 3D extended operator technique relative to the existing ones, we have computed the baryonic two point functions with standard point sources and Jacobi smeared sources. The free parameters of the Jacobi smearing are the number of terms included into the sum $N_{s m}$ and $\kappa_{s m}$ which regulates how strongly the source is spread out [7]

$$
\Psi_{\mathrm{sm}}=\sum_{n=0}^{N_{s m}}\left(\kappa_{s m} \Delta\right)^{n} \Psi_{\mathrm{pnt}}
$$

The parameters have been chosen to be $N_{s m}=50$ and $\kappa_{s m}=0.21$, such that the square of the source radius matches to the one of the 3D fermions. The Jacobi smeared source $\Psi_{s m}$ behaves very differently from the 3D one as can be seen in Fig. 2. The latter has the typical exponential decay in the long distance region, whereas the Jacobi source has a the shape similar to a Gaussian. The different shapes of these two source types give the opportunity to construct operators that differ significantly from one another. Therefore they might be well suited to construct an enlarged basis to be used with variational methods.

Finally, Figs. 3 and 4 show the effective masses of the nucleon and the Omega, respectively, as a function of source sink separation. For both baryons, the 3D extended operators have an excellent 


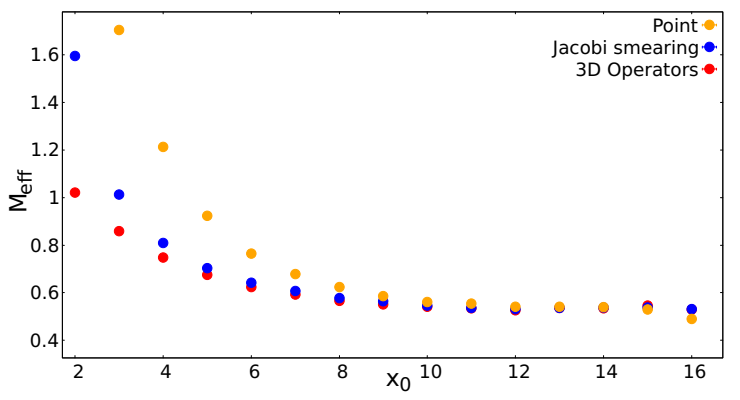

(a) $\hat{p}$ from eq.(2.2)

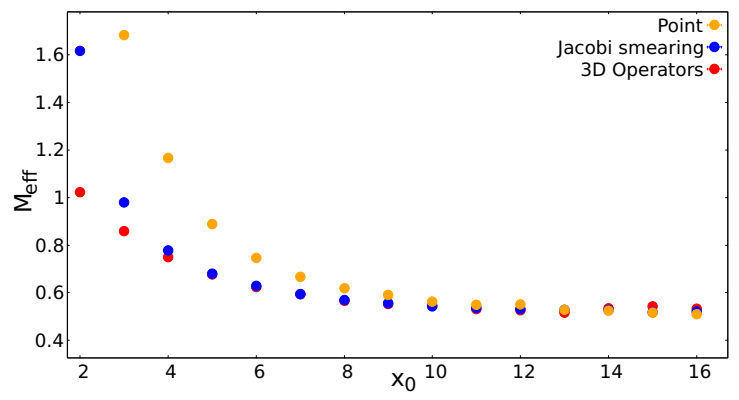

(b) $\tilde{p}$ from eq.(2.2)

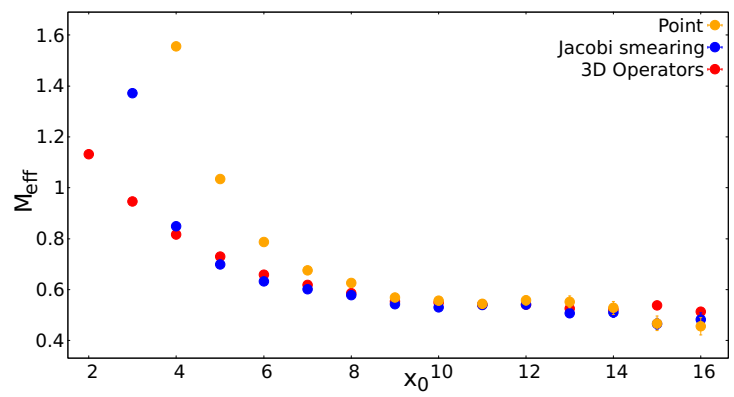

(c) $p$ from eq.(2.3)

Figure 3: Comparison between the effective masses of the nucleon calculated for the 3D extended operators (red) and the Jacobi smearing (blue) and point sources (orange) for the three different nucleon operators of eqs.(2.2) and (2.3). The 3D extended operators have a better suppression of the contributions from the excited states and also the short distance behaviour is much more regular. While similar from about $x_{0}=4 a$ on, the Jacobi smeared sources have a stronger coupling to the excited states at smaller distance.

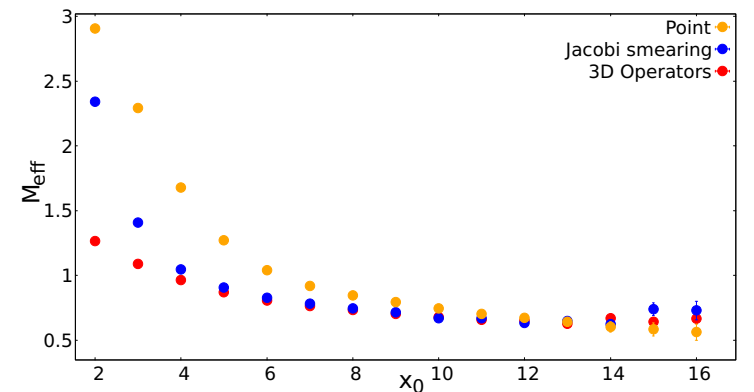

(a) $\Omega^{\left(\frac{1}{2}, 1\right)}$ from eq.(2.4)

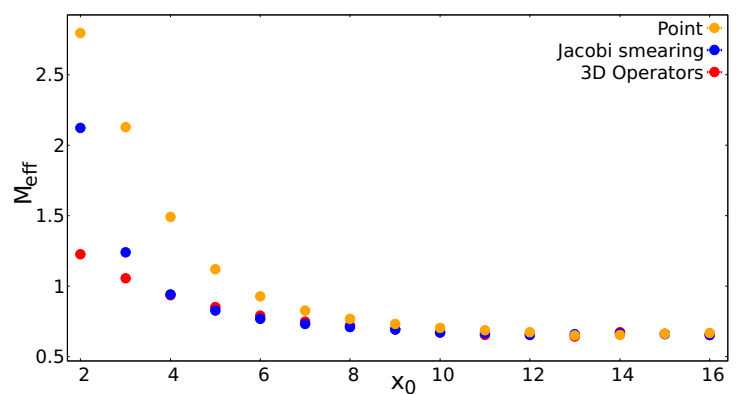

(b) $\Omega^{\left(\frac{3}{2}, 0\right)}$ from eq.(2.4)

Figure 4: The comparison between the effective masses of the Omega calculated for the 3D extended operators (red) and the Jacobi smearing (blue) and point sources (orange). The effective mass is extracted from the two point functions built from the two different Omega operators of eq.(2.4). It is possible to draw similar conclusions to the nucleon case. 
short distance behaviour compared to the other two methods. On the other hand, the plateaux for both, the 3D extended operators and Jacobi smearing, are reached at about the same time and have very similar statistical noise.

\section{Summary}

The 3D fermions introduced in this poster are an interesting alternative to the more standard Jacobi smearing and look promising in increasing the precision in the extraction of the baryon spectrum. In the numerical application, the 3D propagators can be computed with standard iterative techniques for sparse systems. This can make them a computationally efficient choice for the construction of wide sources.

It can be shown that these operators improve the short distance behaviour of two point functions and that they are well behaved under renormalisation. The 3D extended operators therefore allow to have theoretical control while taking the continuum limit. The disappearance of short distance divergences might improve the study of excited states, where a strong signal is needed at short Euclidean times. However a satisfactory understanding of the interplay between the short distance behaviour and excited states can only be achieved through a scaling study, one of the next steps we are planning.

The previous properties together with their difference from the Jacobi smeared sources and the freedom given by the tunable $m_{3 D}$ mass parameter make the 3D extended operators a potentially very interesting addition to the basis used in the GEVP and therefore promise to help in the extraction of the spectrum.

We thank Martin Lüscher for many discussions on the subject.

\section{References}

[1] S. Basak et al., Group-theoretical construction of extended baryon operators in lattice QCD, Phys. Rev. D72 (2005) 094506, [hep-lat/ 0506029 ].

[2] LatTiCe Hadron Physics (LHPC) collaboration, S. Basak et al., Clebsch-Gordan construction of lattice interpolating fields for excited baryons, Phys. Rev. D72 (2005) 074501, [hep-lat / 0508018 ].

[3] R. C. Johnson, Angular momentum on a lattice, Phys. Lett. B114 (1982) 147-151.

[4] M. Bruno et al., Simulation of $Q C D$ with $N_{f}=2+1$ flavors of non-perturbatively improved Wilson fermions, JHEP 02 (2015) 043, [1411.3982].

[5] M. Lüscher and S. Schaefer, Lattice QCD without topology barriers, JHEP 07 (2011) 036, [1105.4749].

[6] M. Bruno, T. Korzec and S. Schaefer, Setting the scale for the CLS $2+1$ flavor ensembles, 1608.08900 .

[7] UKQCD collaboration, C. R. Allton et al., Gauge invariant smearing and matrix correlators using Wilson fermions at Beta $=6.2$, Phys. Rev. D47 (1993) 5128-5137, [hep-lat/930300 9]. 